\title{
THE QUANTUM SCATTERING LIMIT FOR A REGULARIZED WIGNER EQUATION*
}

\author{
BENOITT PERTHAME ${ }^{\dagger}$ AND LENYA RYZHIK ${ }^{\ddagger}$
}

\begin{abstract}
We consider a regularized Wigner equation with an oscillatory kernel, the regularization acts in the space variable to damp high frequencies. The oscillatory kernel is directly derived from the Schrödinger equation with an oscillatory potential. The problem therefore contains three scales, $\varepsilon$ the oscillation length, $\theta$ the regularization parameter, $\delta$ the potential lattice.

We prove that the homogenized limit (as $\varepsilon$ vanishes) of this equation is a scattering equation with discrete jumps. As $\delta$ vanishes, the discrete scattering kernel boils down to a standard regular scattering kernel. As $\theta$ vanishes we recover the quantum scattering operator with collisions preserving energy sphere.
\end{abstract}

Key words. Wigner equation, quantum scattering, weak coupling limit

AMS subject classifications. 35B25, 35B27, 35Q40, 81Q20, 82C40

1. Introduction. The kinetic equation

$$
\frac{\partial \bar{W}}{\partial t}+k \cdot \nabla_{x} \bar{W}=\int_{\mathbb{R}^{d}}|\alpha(p-k)|^{2}[\bar{W}(p)-\bar{W}(k)] \delta\left(\frac{k^{2}-p^{2}}{2}\right) d p .
$$

describes the phase space semi-classical limit of the energy density of the solutions of the Schrödinger equation in the weak coupling limit:

$$
i \varepsilon \frac{\partial \psi_{\varepsilon}}{\partial t}+\varepsilon^{2} \Delta \psi_{\varepsilon}+\sqrt{\varepsilon} V\left(\frac{x}{\varepsilon}\right) \psi_{\varepsilon}=0
$$

as $\varepsilon \rightarrow 0$. Here $x \in \mathbb{R}^{d}$ is the physical space coordinate and $k \in \mathbb{R}^{d}$ is the wave vector. The passage from (1.2) to (1.1) with a spatially homogeneous random potential $V$ has been first proved in $[18,13]$ for a short time interval and later extended to a global in time result in [10]. More precisely, it turns out that $\bar{W}(t, x, k)$ is the weak limit as $\varepsilon \rightarrow 0$ of the Wigner transform of $\psi$ defined as

$$
W_{\varepsilon}(t, x, k)=\int e^{i k \cdot y} \psi_{\varepsilon}\left(t, x+\frac{\varepsilon y}{2}\right) \psi_{\varepsilon}^{*}\left(t, x-\frac{\varepsilon y}{2}\right) \frac{d y}{(2 \pi)^{d}} .
$$

The Wigner transform itself satisfies an evolution equation

$$
\frac{\partial W_{\varepsilon}}{\partial t}+k \cdot \nabla_{x} W_{\varepsilon}=\frac{1}{i \sqrt{\varepsilon}} \int e^{i p \cdot x / \varepsilon}\left[W_{\varepsilon}\left(x, k-\frac{p}{2}\right)-W_{\varepsilon}\left(x, k+\frac{p}{2}\right)\right] \hat{V}(p) \frac{d p}{(2 \pi)^{d}} .
$$

The scattering cross-section $|\alpha(p)|^{2}$ in (1.1) turns out to be the power spectrum of the random potential $V$.

The proofs in $[18,13,10]$ are based on the intricate analysis of the individual contributions of various terms in the Duhamel expansion of (1.2) and are highly technical. The difficulties are intrinsic to the problem as the limit is only weak and

\footnotetext{
*Received September 6, 2004; accepted for publication November 18, 2004.

† Département de Mathématiques et Applications, École Normale Supérieure, CNRS UMR8553, 45 rue d'Ulm, F 75230 Paris cedex 05, France (perthame@dma.ens.fr).

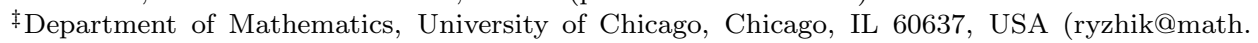
uchicago.edu).
} 
the oscillatory terms are not small in the strong norms. The problem becomes much simpler if the random potential is random in time as well $[2,3,11,16]$ - this introduces an additional mixing that allows to obtain $L^{2}$ estimates on the corrected test functions for (1.4).

The purpose of this paper is to consider a deterministic model where the kinetic limit can be obtained in a straightforward manner. It turns out that this may be achieved by introducing a high-frequency damping in the Wigner equation, replacing (1.4) with

$$
\begin{aligned}
& \frac{\partial W_{\varepsilon}}{\partial t}+k \cdot \nabla_{x} W_{\varepsilon}+\frac{\theta}{\varepsilon}\left(W_{\varepsilon}-\chi_{\varepsilon} \star W_{\varepsilon}\right) \\
= & \frac{1}{i \sqrt{\varepsilon}} \int e^{i p \cdot x / \varepsilon}\left[W_{\varepsilon}\left(x, k-\frac{p}{2}\right)-W_{\varepsilon}\left(x, k+\frac{p}{2}\right)\right] \hat{V}(p) \frac{d p}{(2 \pi)^{d}},
\end{aligned}
$$

with a positive function $\chi_{\varepsilon}=\varepsilon^{-d} \chi(x / \varepsilon)$ such that $\int \chi(x) d x=1$. The regularization parameter $\theta \ll 1$ is small. Heuristically, the last term on the left side of (1.5) is absorbing for the high frequency component as $\chi_{\varepsilon} \star W_{\varepsilon}^{h f} \approx 0$ while it is not damping the low frequencies of $W_{\varepsilon}$, since $\chi_{\varepsilon} \star W_{\varepsilon}^{l f} \approx W_{\varepsilon}^{l f}$ for the low frequency part of $W_{\varepsilon}$. This is also reflected in the energy balance

$$
\frac{1}{2} \frac{d}{d t} \int|W(t, x, k)|^{2} d x d k=-\frac{\theta}{\varepsilon} \int(1-\hat{\chi}(\varepsilon p))|\hat{W}(t, p, k)|^{2} d p d k \leq 0 .
$$

Hence, the purpose of the weak high frequency damping is to capture correctly only the low frequency behavior while getting rid of the high frequency oscillations. This leads to the strong $L^{2}$-convergence of the solution of (1.5) to the solution of a kinetic equation as the high frequency oscillations are absent in the limit.

The potential $V$ in (1.5) is not required to be random or periodic: the only requirement is that its Fourier transform has a non-trivial singular part: see (2.4) below. This is another interesting aspect of the current set-up: the regularized Wigner equation may be homogenized in a very general setting with almost no underlying small-scale structure, such as periodicity or statistical homogeneity, assumed.

On the other hand, the derivation of a scattering equation from the true Wigner equation with a given potential is certainly impossible in a general deterministic framework. This is because of some inconsistency in the cancellations in the potential that are involved to produce the coefficient $\alpha^{2}$ that depends upon $V$ in a quadratic way, a phenomena that has been pointed out in [8]. Introduction of a regularization allows us to get forward with several steps which are based on three different limits. Firstly the homogenization parameter $\varepsilon$ vanishes, secondly the potential pseudo period lattice, denoted by $\delta$ below vanishes and thirdly the regularization parameter $\theta$ vanishes. We note that the final result of the three sequential limits is exactly the same kinetic equation (1.1) with an appropriately defined function $\alpha(p)$. Two comments are in order: first, the final kinetic equation is completely independent of the choice of the regularization function $\chi(x)$. Second, only the singular part of the measure-valued Fourier transform $\hat{V}(p)$ contributes to the scattering cross-section.

We note that the result we prove below, the strong convergence to the homogenized limit, is certainly impossible for the unregularized Wigner equation because it preserves the $L^{2}$ norm of the solution, while the scattering equation does not.

There are other ways to obtain a non-trivial semi-classical limit with a nonuniform potential. One way is a different scaling: we refer to [14] for the derivation of the standard Liouville equation for a fixed slowly varying potential, and to [1, 12] 
for the semi-classical limit in a rapidly oscillating periodic potential. None of them produce scattering but rather a modification of the bi-characteristics. Another way mentioned above to produce a scattering term is to introduce random potentials as performed in $[18,13,10]$. The capability of a deterministic model to produce a scattering operator in the limit is more recent: see $[7,15]$ for two different formalisms (a simple homogeneous box without transport in the limit, or scattering localized at a single point). In the regime and method of Born series, the results have been pushed forward in [5], [6].

Our formalism allows us to use different methods that rely on the homogenization methods as presented in $[4,9]$ for instance, that is, building a multi-scale expansion

$$
W_{\varepsilon}=\bar{W}+\sqrt{\varepsilon} W_{1}\left(t, x, \frac{x}{\varepsilon}, k\right)+\varepsilon W_{2}\left(t, x, \frac{x}{\varepsilon}, k\right)+\ldots
$$

Here again the regularized equation allows us to make sense of the expansion. The specific difficulty is that the corrector equation for $W_{1}, W_{2}$ is ill-posed without regularization in our framework.

We recall that the general formalism behind the transition wave $\rightarrow$ Wigner $\rightarrow$ scattering is much more general than for the Schrödinger equation, we refer to [17] for a general presentation of this subject. However, there are no rigorous results on the passage to the kinetic limit available for time-independent systems other than the Schrödinger equation. On the other hand, the method of the present paper should extend to the regularized versions of the Wigner equations that correspond to, say, the acoustic wave equations, without major difficulties.

The organization of the paper is as follows. We present in Section 2 in detail our model and the various results. We insist in particular on the interplay and relative size of the various parameters that arise here. The third section is devoted to the construction of the correctors and to preliminary estimates. As usual, the scattering equation arises as a solvability condition in order to be able to build the correctors. However, the solvability condition is not completely standard. The fourth section furnishes the technical estimates on the remainder and proves the main theorem (the limit $\varepsilon \rightarrow 0$ ). The last section deals with the easier limits on the two additional parameters of the problem.

Acknowledgment. This work has been performed while LR was visiting ENS, Paris. He thanks ENS for its hospitality. LR's work was also supported in part by NSF grant DMS-0203537.

2. The main results. We consider a regularized Wigner equation

$$
\begin{gathered}
\frac{\partial W_{\varepsilon}}{\partial t}+k \cdot \nabla_{x} W_{\varepsilon}+\frac{\theta}{\varepsilon}\left(W_{\varepsilon}-\chi_{\varepsilon} \star W_{\varepsilon}\right)=\mathcal{L}_{\varepsilon} W_{\varepsilon}, \quad t \geq 0, x \in \mathbb{R}^{d}, k \in \mathbb{R}^{d}, \\
\mathcal{L}_{\varepsilon} f(x, k)=\frac{1}{i \sqrt{\varepsilon}} \int e^{i p \cdot x / \varepsilon}\left[f\left(x, k-\frac{p}{2}\right)-f\left(x, k+\frac{p}{2}\right)\right] \hat{V}(p) \frac{d p}{(2 \pi)^{d}} .
\end{gathered}
$$

Throughout the paper the notation $\hat{f}$ represents the Fourier transform in the $x$ variable

$$
\hat{f}(p)=\int e^{-i p \cdot x} f(x) d x,
$$

and the inverse Fourier transform is then

$$
f(x)=\int e^{i p \cdot x} \hat{f}(p) \frac{d p}{(2 \pi)^{d}} .
$$


The function $\chi_{\varepsilon}(x)=\frac{1}{\varepsilon^{d}} \chi\left(\frac{x}{\varepsilon}\right)$ in (2.1) with $\chi \in \mathcal{S}\left(\mathbb{R}^{d}\right)$ (the Schwartz space) and $\chi(x)=\chi(|x|) \geq 0$ radially symmetric, and normalized so that

$$
\int_{\mathbb{R}^{d}} \chi(x) d x=1
$$

Henceforth, $\hat{\chi} \in \mathcal{S}\left(\mathbb{R}^{d}\right)$ satisfies

$$
\hat{\chi} \in \mathbb{R}, \quad|\hat{\chi}(p)|<1 \text { for } p \neq 0, \quad \hat{\chi}(0)=1 .
$$

The parameter $\theta$ is small but fixed - we may allow $\theta$ to depend on $\varepsilon$ so that $\theta \gg \varepsilon$ but we do not pursue this issue here for the sake of clarity of presentation. The last term on the left side of (2.1) is regularizing in $L^{2}$, that is:

$$
\frac{1}{2} \frac{d}{d t} \int\left|W_{\varepsilon}(t, x, k)\right|^{2} d x d k=-\frac{\theta}{\varepsilon} \int|1-\hat{\chi}(\varepsilon p)|^{2}\left|\hat{W}_{\varepsilon}(p, k)\right|^{2} \frac{d p d k}{(2 \pi)^{d}} .
$$

The energy balance (2.3) shows that the effect of the regularization is damping of the high frequencies. This allows us to show the strong convergence of the solution of (2.1) to the solution of a kinetic equation in the limit $\varepsilon \rightarrow 0$. The regularization allows us to make the formal asymptotic expansions rigorous and circumvent dealing with the weak convergence.

We assume that the Fourier transform of the potential $V(x)$ has the form

$$
\hat{V}(p)=\sum_{j=1}^{\infty} \alpha_{j}\left[\delta\left(p-p_{j}\right)+\delta\left(p+p_{j}\right)\right]+\hat{\Phi}(p)
$$

with the real Fourier coefficients $\alpha_{j} \in \mathbb{R}$ and $\hat{\Phi}(p)$ that is smooth, sufficiently rapidly decaying and with $\hat{\Phi}(0)=0$. We also assume that the sequence $\alpha_{j}$ satisfies the following conditions:

$$
\sum_{j=1}^{\infty} \frac{\left|\alpha_{j}\right|}{\left|1-\hat{\chi}\left(p_{j}\right)\right|}<+\infty
$$

and

$$
\sum_{j, l=1}^{\infty} \frac{\left|\alpha_{j}\right|\left|\alpha_{l}\right|}{\left|1-\hat{\chi}\left(p_{l}\right)\right|\left|1-\hat{\chi}\left(p_{j}+p_{l}\right)\right|}+\sum_{j \neq l} \frac{\left|\alpha_{j}\right|\left|\alpha_{l}\right|}{\left.\left|1-\hat{\chi}\left(p_{l}\right)\right| \mid 1-\hat{\chi}\left(p_{j}-p_{l}\right)\right) \mid}<+\infty .
$$

Recall that $\hat{\chi}(0)=1$ so that $(2.5)$ means that $\hat{V}(p)$ is not singular at $p=0$ : oscillations are not concentrated at the zero wave number.

These conditions are satisfied if, for instance, $\alpha_{j} \in l^{1}$ and the wave vectors $p_{j}$ are non-resonant: there exists $\omega_{0}>0$ so that

$$
\left|p_{j}\right| \geq \omega_{0}>0, \quad\left|p_{j} \pm p_{l}\right| \geq \omega_{0} \text { for } j \neq l .
$$

On the other hand, (2.5) implies that $\alpha_{j} \in l^{1}$ and thus the potential $\left.V(x)\right)$ satisfies

$$
|V(x)| \leq \int|\hat{V}(p)| d p<+\infty .
$$


It follows that the operator $\mathcal{L}_{\varepsilon}$ is uniformly bounded from $L^{2}\left(\mathbb{R}^{d} \times \mathbb{R}^{d}\right)$ into itself and the existence theory for (2.1)-(2.2) is thus standard.

We define the scattering kernel

$$
K_{\theta}(k, p)=\frac{1}{(2 \pi)^{d}} \frac{2 \theta(1-\hat{\chi}(p))}{\theta^{2}(1-\hat{\chi}(p))^{2}+\left(\left(k+\frac{p}{2}\right) \cdot p\right)^{2}},
$$

and use the convention that for $j \leq-1, p_{j}=-p_{-j}$. Then we have the following theorem which shows that only the singular (oscillatory) component of the potential affects the limit.

Theorem 2.1. Let the initial data $W_{\varepsilon}(0, x, k)=W_{0}(x, k)$ for (2.1) belong to $L^{2}\left(\mathbb{R}^{d} \times \mathbb{R}^{d}\right)$ and assume (2.4), (2.5) and (2.6) on the potential $V(x)$ and the regularization function $\chi(x)$. Then the operator $\mathcal{L}_{\varepsilon}$ is uniformly bounded on $L^{2}\left(\mathbb{R}^{d} \times \mathbb{R}^{d}\right)$ and the solution of (2.1) converges in $C\left([0, T] ; L^{2}\left(\mathbb{R}^{d} \times \mathbb{R}^{d}\right)\right)$ to the solution of the kinetic equation

$$
\frac{\partial \bar{W}}{\partial t}+k \cdot \nabla_{x} \bar{W}=\sum_{j \in \mathbb{Z}^{*}}\left|\alpha_{j}\right|^{2} K_{\theta}\left(k, p_{j}\right)\left[\bar{W}\left(k+p_{j}\right)-\bar{W}(k)\right]
$$

with the initial data $\bar{W}(0, x, k)=W_{0}(x, k)$.

Note that the scattering kernel $K_{\theta}\left(k, p_{j}\right)$ is positive and (2.9) is a kinetic equation that may be given a probabilistic interpretation. Physically, the scattering crosssection depends only on the singular part of the potential because a weak $O(\sqrt{\varepsilon})$ localized potential due to $\hat{\Phi}(p)$ in (2.4) does not affect the wave energy propagation over long distances, as opposed to the potential due to the singular part of the spectrum that is "present everywhere".

Let us now assume that we are given a family of potentials $V^{\delta}(x)$ of the form (2.4), parametrized by a parameter $\delta>0$, such that, uniformly,

$$
\sum_{j=1}^{\infty}\left|\alpha_{j}^{\delta}\right|^{2} \frac{1}{1-\hat{\chi}\left(p_{j}^{\delta}\right)}<\infty
$$

For instance the wave vectors $p_{j}^{\delta}$ may be picked so that there is exactly one $p_{j}$ in each cube of a cubic lattice in $\mathbb{R}_{+}^{d}=\left\{q=\left(q_{1}, \ldots, q_{d}\right) \in \mathbb{R}^{d}: q_{1}>0\right\}$ with the cube side $\delta \ll 1$, while the amplitudes are scaled so that $\alpha_{j}^{\delta}=\delta^{d / 2} \alpha\left(p_{j}^{\delta}\right)$ for a smooth function $\alpha(p)$. Then the scattering term on the right side of (2.9) has the form

$$
\mathcal{K}_{\theta}^{\delta} \bar{W}(k)=\delta^{d} \sum_{j \in \mathbb{Z}^{*}}\left|\alpha\left(p_{j}^{\delta}\right)\right|^{2} K_{\theta}\left(k, p_{j}^{\delta}\right)\left[\bar{W}\left(k+p_{j}^{\delta}\right)-\bar{W}(k)\right]
$$

that is a Riemann sum of

$\int|\alpha(p)|^{2} K_{\theta}(k, p)[\bar{W}(k+p)-\bar{W}(k)] d p=\int|\alpha(p-k)|^{2} K_{\theta}(k, p-k)[\bar{W}(p)-\bar{W}(k)] d p$,

with

$$
\int|\alpha(p)|^{2} \frac{1}{1-\hat{\chi}(p)} d p<\infty
$$

We have the following convergence result. 
TheOREM 2.2. Let the initial data $W_{0}(x, k)$ for (2.9) belong to $L^{2}\left(\mathbb{R}^{d} \times \mathbb{R}^{d}\right)$ and make the above assumptions (2.10), (2.11) on the distribution of the points $p_{j}^{\delta}$ and amplitudes $\alpha_{j}^{\delta}$. Then, the operator $\mathcal{K}_{\theta}^{\delta}$ is uniformly bounded in $L^{2}\left(\mathbb{R}^{d}\right)$ as $\delta$ vanishes and the solution $\bar{W}_{\theta}^{\delta}$ of (2.9) converges in $C\left([0, T] ; L^{2}\left(\mathbb{R}^{d} \times \mathbb{R}^{d}\right)\right)$ to the solution of the kinetic equation

$$
\frac{\partial \bar{U}}{\partial t}+k \cdot \nabla_{x} \bar{U}=\int_{\mathbb{R}^{d}}|\alpha(p-k)|^{2} K_{\theta}(k, p-k)[\bar{U}(p)-\bar{U}(k)] d p
$$

with the initial data $\bar{U}(0, x, k)=W_{0}(x, k)$.

Equation (2.12) is now a continuous scattering equation but it allows interaction of waves with different frequencies $\omega=k^{2} / 2$, unlike the kinetic equation (1.1) which preserves the energy sphere. The final observation is that the scattering kernel $K_{\theta}(k, p-k)$ converges as $\theta \rightarrow 0$ :

$$
\begin{aligned}
P_{\theta}(k, p) & =|\alpha(p-k)|^{2} K_{\theta}(k, p-k) \\
& =|\alpha(p-k)|^{2} 2 \theta(1-\hat{\chi}(p-k))\left\{\theta^{2}(1-\hat{\chi}(p-k))^{2}+\left(\frac{p^{2}-k^{2}}{2}\right)^{2}\right\}^{-1} \\
& \rightarrow \frac{2 \pi|\alpha(p-k)|^{2}}{1-\hat{\chi}(p-k)} \delta\left(\frac{p^{2}-k^{2}}{2(1-\hat{\chi}(p-k))}\right) \\
& =2 \pi|\alpha(p-k)|^{2} \delta\left(\frac{p^{2}-k^{2}}{2}\right) .
\end{aligned}
$$

This calculation requires an extra assumption in order to manipulate the operator

$$
\mathcal{P}_{\theta} U(k)=\int|\alpha(p-k)|^{2} K_{\theta}(k, p-k)[U(p)-U(k)] d p,
$$

namely

$$
M_{\alpha}:=\int_{S^{d-1}} \sup _{r>0} r^{d-2}|\alpha(r \omega)|^{2} d \omega<\infty
$$

This implies our last result.

Theorem 2.3. Let the initial data $W_{0}(x, k)$ for (2.12) belong to $L^{2}\left(\mathbb{R}^{d} \times \mathbb{R}^{d}\right)$ and assume (2.14) on the scattering function $\alpha(p)$ and the bound of Lemma 5.1 on $\chi$. Then, the operator $\mathcal{P}_{\theta}$ is uniformly bounded in $L^{2}\left(\mathbb{R}^{d}\right)$ and the solution $\bar{U}_{\theta}$ of (2.12) converges in $C\left([0, T] ; L^{2}\left(\mathbb{R}^{d} \times \mathbb{R}^{d}\right)\right)$, as $\theta \rightarrow 0$, to the solution of the kinetic equation

$$
\frac{\partial \bar{Z}}{\partial t}+k \cdot \nabla_{x} \bar{Z}=\int_{\mathbb{R}^{d}}|\alpha(p-k)|^{2}[\bar{Z}(p)-\bar{Z}(k)] \delta\left(\frac{k^{2}-p^{2}}{2}\right) d p
$$

with the initial data $\bar{W}(0, x, k)=W_{0}(x, k)$.

Note that the final equation (2.15) is independent from the regularization function $\chi(p)$ and is nothing but the transport equation (1.1) with an appropriately defined scattering cross-section $\alpha(\cdot)$. 
3. Proof of Theorem 2.1: the formal expansion. In order to make the proof less cumbersome we will assume that the smooth component $\hat{\Phi}(p)$ in (2.4) vanishes. Its presence does not modify the proof in any significant way but makes the calculations somewhat longer.

We will construct an expansion

$$
W_{\varepsilon}(t, x, k)=\bar{W}(t, x, k)+\sqrt{\varepsilon} W_{1}^{\varepsilon}+\varepsilon W_{2}^{\varepsilon}+R_{\varepsilon} .
$$

Here $\bar{W}(t, x, k)$ is the solution of the kinetic equation (2.9). The two correctors $W_{j}^{\varepsilon}(t, x, k)=W_{j}(t, x, x / \varepsilon, k), j=1,2$, are bounded in $L^{2}\left(\mathbb{R}_{x}^{d} \times \mathbb{R}_{k}^{d}\right)$ and will be constructed explicitly so as to make the remainder $R_{\varepsilon}$ have $L^{2}$-norm of order $O(\sqrt{\varepsilon})$. We note that the functions $W_{1,2}(t, x, z, k)$ do not decay in the fast variable $z=x / \varepsilon$ but only in the macroscopic variables $x$ and $k$-this is similar to the usual periodic homogenization $[4,9]$.

The construction of the functions $W_{1,2}(t, x, z, k)$ is similar to that in the case when the potential $V$ is random and time-dependent $[2,3,11]$. The main difference with the present case is in the way the second corrector is defined and bounded.

The first corrector. The correctors are built as follows. The first corrector $W_{1}$ cancels the terms that are formally of the order $\varepsilon^{-1 / 2}$ when we insert the formal expansion (3.1) into the evolution equation (2.1) for $W_{\varepsilon}$. It satisfies

$$
\begin{aligned}
& k \cdot \nabla_{z} W_{1}+\theta\left(W_{1}-\int \chi(y) W_{1}(t, x, z-y) d y\right) \\
= & \frac{1}{i} \int e^{i p \cdot z}\left[\bar{W}\left(t, x, k-\frac{p}{2}\right)-\bar{W}\left(t, x, k+\frac{p}{2}\right)\right] \frac{\hat{V}(p) d p}{(2 \pi)^{d}} .
\end{aligned}
$$

Taking the Fourier transform in $z$, we obtain

$$
[\theta(1-\hat{\chi}(p))+i k \cdot p] \hat{W}_{1}(t, x, p, k)=\frac{1}{i} \hat{V}(p)\left[\bar{W}\left(t, x, k-\frac{p}{2}\right)-\bar{W}\left(t, x, k+\frac{p}{2}\right)\right],
$$

where $p$ is the dual Fourier variable to $z$. Hence, the first term in the expansion (3.1) may be written as

$$
W_{1}^{\varepsilon}(t, x, k)=\int e^{i p \cdot x / \varepsilon} \frac{\hat{V}(p)\left[\bar{W}\left(t, x, k-\frac{p}{2}\right)-\bar{W}\left(t, x, k+\frac{p}{2}\right)\right]}{i(\theta(1-\hat{\chi}(p))+i k \cdot p)} \frac{d p}{(2 \pi)^{d}} .
$$

We observe that

$$
\begin{aligned}
& \left\|W_{1}^{\varepsilon}(t)\right\|_{L^{2}\left(\mathbb{R}^{2 d}\right)} \\
\leq & \frac{1}{\theta} \int \frac{|\hat{V}(p)|}{|1-\hat{\chi}(p)|}\left[\left\|\bar{W}\left(t, x, k+\frac{p}{2}\right)\right\|_{L^{2}\left(\mathbb{R}^{2 d}\right)}+\left\|\bar{W}\left(t, x, k-\frac{q}{2}\right)\right\|_{L^{2}\left(\mathbb{R}^{2 d}\right)}\right] \frac{d p}{(2 \pi)^{d}} \\
\leq & \frac{C}{\theta} \int \frac{|\hat{V}(p)| d p}{|1-\hat{\chi}(p)|}\|\bar{W}(t)\|_{L^{2}\left(\mathbb{R}^{2 d}\right)} \leq C \sum_{j=1}^{\infty} \frac{\left|\alpha_{j}\right|}{\left|1-\hat{\chi}\left(p_{j}\right)\right|}\left\|W_{0}\right\|_{L^{2}\left(\mathbb{R}^{2 d}\right)},
\end{aligned}
$$

and it follows from the assumption (2.5) in Theorem 2.1 that the $L^{2}$-norm of $W_{1}^{\varepsilon}$ is bounded. 
The second corrector. The corrector $W_{2}(t, x, z, k)$ should cancel terms of order one when we insert the expansion (3.1) into (2.1). It satisfies

$$
\begin{gathered}
k \cdot \nabla_{z} W_{2}+\theta\left(W_{2}-\int \chi(y) W_{2}(t, x, z-y) d y\right)=-\left(\frac{\partial \bar{W}}{\partial t}+k \cdot \nabla_{x} \bar{W}\right) \\
+\frac{1}{i} \int e^{i p \cdot z}\left[W_{1}\left(t, x, k-\frac{p}{2}\right)-W_{1}\left(t, x, k+\frac{p}{2}\right)\right] \frac{\hat{V}(p) d p}{(2 \pi)^{d}} .
\end{gathered}
$$

The integral on the second line above may be re-written, using expression (3.4) for $W_{1}$ (with $x / \varepsilon$ replaced by $z$ ), as

$$
\begin{aligned}
& \frac{1}{i} \int e^{i p \cdot z}\left[W_{1}\left(t, x, k-\frac{p}{2}\right)-W_{1}\left(t, x, k+\frac{p}{2}\right)\right] \frac{\hat{V}(p) d p}{(2 \pi)^{d}}=\frac{1}{i} \int e^{i(p+q) \cdot z} \hat{V}(p) \hat{V}(q) \\
& \quad \times\left[\frac{\bar{W}\left(k-\frac{p}{2}-\frac{q}{2}\right)-\bar{W}\left(k-\frac{p}{2}+\frac{q}{2}\right)}{i\left(\theta(1-\hat{\chi}(q))+i\left(k-\frac{p}{2}\right) \cdot q\right)}-\frac{\bar{W}\left(k+\frac{p}{2}-\frac{q}{2}\right)-\bar{W}\left(k+\frac{p}{2}+\frac{q}{2}\right)}{i\left(\theta(1-\hat{\chi}(q))+i\left(k+\frac{p}{2}\right) \cdot q\right)}\right] \frac{d p d q}{(2 \pi)^{2 d}} .
\end{aligned}
$$

This may be further transformed as

$$
\begin{aligned}
& \int e^{i(p+q) \cdot z} \hat{V}(p) \hat{V}(q)\left[\frac{\bar{W}\left(k-\frac{p}{2}+\frac{q}{2}\right)-\bar{W}\left(k-\frac{p}{2}-\frac{q}{2}\right)}{\theta(1-\hat{\chi}(q))+i\left(k-\frac{p}{2}\right) \cdot q}+\frac{\bar{W}\left(k+\frac{p}{2}-\frac{q}{2}\right)-\bar{W}\left(k+\frac{p}{2}+\frac{q}{2}\right)}{\theta(1-\hat{\chi}(q))+i\left(k+\frac{p}{2}\right) \cdot q}\right] \frac{d p d q}{(2 \pi)^{2 d}} \\
& =\int e^{i p \cdot z} \hat{V}(p-q) \hat{V}(q)\left[\frac{\bar{W}\left(k-\frac{p}{2}+q\right)-\bar{W}\left(k-\frac{p}{2}\right)}{\theta(1-\hat{\chi}(q))+i\left(k-\frac{p-q}{2}\right) \cdot q}+\frac{W_{0}\left(k+\frac{p}{2}-q\right)-W_{0}\left(k+\frac{p}{2}\right)}{\theta(1-\hat{\chi}(q))+i\left(k+\frac{p-q}{2}\right) \cdot q}\right] \frac{d p d q}{(2 \pi)^{2 d}} \\
& =\int e^{i p \cdot z} G(p) \frac{d p}{(2 \pi)^{d}}
\end{aligned}
$$

where the function $G(t, x, k, p)$ is defined by

$$
\begin{aligned}
G(p)=\int \hat{V}(p-q) \hat{V}(q)\left[\frac{\bar{W}\left(k-\frac{p}{2}+q\right)-\bar{W}\left(k-\frac{p}{2}\right)}{\theta(1-\hat{\chi}(q))+i\left(k-\frac{p-q}{2}\right) \cdot q}\right. \\
\left.\quad+\frac{\bar{W}\left(k+\frac{p}{2}-q\right)-\bar{W}\left(k+\frac{p}{2}\right)}{\theta(1-\hat{\chi}(q))+i\left(k+\frac{p-q}{2}\right) \cdot q}\right] \frac{d q}{(2 \pi)^{d}} .
\end{aligned}
$$

We may re-write (3.5) as

$$
\begin{aligned}
& k \cdot \nabla_{z} W_{2}+\theta\left(W_{2}-\int \chi(y) W_{2}(t, x, z-y) d y\right) \\
& =-\left(\frac{\partial \bar{W}}{\partial t}+k \cdot \nabla_{x} \bar{W}\right)+\int e^{i p \cdot z} G(p) \frac{d p}{(2 \pi)^{d}} .
\end{aligned}
$$

After Fourier transform in $z$ we arrive at

$$
[\theta(1-\hat{\chi}(p))+i k \cdot p] \hat{W}_{2}(t, x, p, k)=-\left(\frac{\partial \bar{W}}{\partial t}+k \cdot \nabla_{x} \bar{W}\right) \delta(p)+G(p) .
$$

Hence, in order for $W_{2}(t, x, z, k)$ to be uniformly bounded in $z$, we have to require that the distribution $G(p)$ defined by (3.6) has the form

$$
G(t, x, p, k)=\left(\frac{\partial \bar{W}}{\partial t}+k \cdot \nabla_{x} \bar{W}\right) \delta(p)+B(t, x, k, p),
$$

where $B(t, x, k, p)$ is sufficiently regular at $p=0$. The matching of the $\delta(p)$ contributions on the two sides of (3.8) gives rise to the kinetic equation (2.9) for $\bar{W}$. 
The transport equation as the solvability condition. In order to see how the equation (2.9) for $\bar{W}$ arises, we insert expression (2.4) for $\hat{V}(p)$ into (3.6) and obtain that the distribution $G(p)$ has the form (recall that we have assumed for simplicity that $\hat{\Phi}(p)=0$ in (2.4))

$$
\begin{aligned}
G(p)= & \sum_{j, l} \int \alpha_{j} \alpha_{l}\left[\delta\left(p-q-p_{j}\right)+\delta\left(p-q+p_{j}\right)\right]\left[\delta\left(q-p_{l}\right)+\delta\left(q+p_{l}\right)\right] \\
& \times\left[\frac{\bar{W}\left(k-\frac{p}{2}+q\right)-\bar{W}\left(k-\frac{p}{2}\right)}{\theta(1-\hat{\chi}(q))+i\left(k-\frac{p-q}{2}\right) \cdot q}+\frac{\bar{W}\left(k+\frac{p}{2}-q\right)-\bar{W}\left(k+\frac{p}{2}\right)}{\theta(1-\hat{\chi}(q))+i\left(k+\frac{p-q}{2}\right) \cdot q}\right] \frac{d q}{(2 \pi)^{d}} \\
= & G_{11}+G_{21}+G_{12}+G_{22} .
\end{aligned}
$$

The four terms above arise from the cross-products of the delta functions. The term $G_{11}$ that comes from $\delta\left(p-p_{j}\right) \delta\left(q-p_{l}\right)$ has the form

$$
\begin{aligned}
G_{11}(p)= & \sum_{j, l} \frac{\alpha_{j} \alpha_{l}}{(2 \pi)^{d}}\left[\frac{\bar{W}\left(k-\frac{p_{j}-p_{l}}{2}\right)-\bar{W}\left(k-\frac{p_{j}+p_{l}}{2}\right)}{\theta\left(1-\hat{\chi}\left(p_{l}\right)\right)+i\left(k-\frac{p_{j}}{2}\right) \cdot p_{l}}+\frac{\bar{W}\left(k+\frac{p_{j}-p_{l}}{2}\right)-\bar{W}\left(k+\frac{p_{j}+p_{l}}{2}\right)}{\theta\left(1-\hat{\chi}\left(p_{l}\right)\right)+i\left(k+\frac{p_{j}}{2}\right) \cdot p_{l}}\right] \\
& \times \delta\left(p-\left(p_{j}+p_{l}\right)\right)=\sum_{j, l} \alpha_{j} \alpha_{l} s_{j l}(t, x, k) \delta\left(p-\left(p_{j}+p_{l}\right)\right) .
\end{aligned}
$$

The contribution $G_{21}$ comes from $\delta\left(p+p_{j}\right) \delta\left(q-p_{l}\right)$, so that

$$
\begin{aligned}
G_{21}(p)= & \sum_{j, l} \frac{\alpha_{j} \alpha_{l}}{(2 \pi)^{d}}\left[\frac{\bar{W}\left(k+\frac{p_{j}+p_{l}}{2}\right)-\bar{W}\left(k-\frac{p_{l}-p_{j}}{2}\right)}{\theta\left(1-\hat{\chi}\left(p_{l}\right)\right)+i\left(k+\frac{p_{j}}{2}\right) \cdot p_{l}}+\frac{\bar{W}\left(k-\frac{p_{j}+p_{l}}{2}\right)-\bar{W}\left(k+\frac{p_{l}-p_{j}}{2}\right)}{\theta\left(1-\hat{\chi}\left(p_{l}\right)\right)+i\left(k-\frac{p_{j}}{2}\right) \cdot p_{l}}\right] \\
& \times \delta\left(p-\left(p_{l}-p_{j}\right)\right)=\sum_{j, l} \alpha_{j} \alpha_{l} r_{j l}(t, x, k) \delta\left(p-\left(p_{l}-p_{j}\right)\right) .
\end{aligned}
$$

The third term $G_{12}$ comes from $\delta\left(p-p_{j}\right) \delta\left(q+p_{l}\right)$, and is given by

$$
\begin{aligned}
G_{12}(p)= & \sum_{j, l} \frac{\alpha_{j} \alpha_{l}}{(2 \pi)^{d}}\left[\frac{\bar{W}\left(k-\frac{p_{j}+p_{l}}{2}\right)-\bar{W}\left(k-\frac{p_{j}-p_{l}}{2}\right)}{\theta\left(1-\hat{\chi}\left(-p_{l}\right)\right)-i\left(k-\frac{p_{j}}{2}\right) \cdot p_{l}}+\frac{\bar{W}\left(k+\frac{p_{j}+p_{l}}{2}\right)-\bar{W}\left(k+\frac{p_{j}-p_{l}}{2}\right)}{\theta\left(1-\hat{\chi}\left(-p_{l}\right)\right)-i\left(k+\frac{p_{j}}{2}\right) \cdot p_{l}}\right] \\
& \times \delta\left(p-\left(p_{j}-p_{l}\right)\right)=\sum_{j, l} \alpha_{j} \alpha_{l} u_{j l}(t, x, k) \delta\left(p-\left(p_{j}-p_{l}\right)\right) .
\end{aligned}
$$

The last term $G_{22}$ arises from $\delta\left(p+p_{j}\right) \delta\left(q+p_{l}\right)$, so that

$$
\begin{aligned}
G_{22}(p)= & \sum_{j, l} \frac{\alpha_{j} \alpha_{l}}{(2 \pi)^{d}}\left[\frac{\bar{W}\left(k+\frac{p_{j}-p_{l}}{2}\right)-\bar{W}\left(k+\frac{p_{j}+p_{l}}{2}\right)}{\theta\left(1-\hat{\chi}\left(-p_{l}\right)\right)-i\left(k+\frac{p_{j}}{2}\right) \cdot p_{l}}+\frac{\bar{W}\left(k-\frac{p_{j}-p_{l}}{2}\right)-\bar{W}\left(k-\frac{p_{j}+p_{l}}{2}\right)}{\theta\left(1-\hat{\chi}\left(-p_{l}\right)\right)-i\left(k-\frac{p_{j}}{2}\right) \cdot p_{l}}\right] \\
& \times \delta\left(p+p_{j}+p_{l}\right)=\sum_{j, l} \alpha_{j} \alpha_{l} q_{j l}(t, x, k) \delta\left(p+p_{j}+p_{l}\right) .
\end{aligned}
$$


Hence, the distribution $G(t, x, p, k)$ has the from

$$
\begin{aligned}
G(t, x, p, k) \\
=\sum_{j, l} \alpha_{j} \alpha_{l}\left[s_{j l}(t, x, k) \delta\left(p-\left(p_{j}+p_{l}\right)\right)+r_{j l}(t, x, k) \delta\left(p-\left(p_{l}-p_{j}\right)\right.\right. \\
\left.\quad+u_{j l}(t, x, k) \delta\left(p-\left(p_{j}-p_{l}\right)\right)+q_{j l}(t, x, k) \delta\left(p+p_{j}+p_{l}\right)\right] \\
=G_{0}(t, x, k) \delta(p)+\sum_{j, l} \alpha_{j} \alpha_{l}\left[s_{j l}(t, x, k) \delta\left(p-\left(p_{j}+p_{l}\right)\right)+q_{j l}(t, x, k) \delta\left(p+p_{j}+p_{l}\right)\right] \\
\quad+\sum_{j \neq l} \alpha_{j} \alpha_{l}\left[r_{j l}(t, x, k) \delta\left(p-\left(p_{l}-p_{j}\right)+u_{j l}(t, x, k) \delta\left(p-\left(p_{j}-p_{l}\right)\right)\right] .\right.
\end{aligned}
$$

The explicit definitions of the coefficients $s_{j l}, r_{j l}, u_{j l}$ and $q_{j l}$ above follow from the expressions (3.9)-(3.12) for $G_{m n}, m, n=1,2$. Note that (3.13) realizes the decomposition (3.8) of $G(p)$ into a delta-function part at $p=0$ and another component that is regular at $p=0$ (in our particular case, as we have assumed that $\hat{\Phi}(p)=0$ in (2.4), this part vanishes at $p=0)$. In particular, we have an explicit expression for $G_{0}(p)$ :

$$
\begin{aligned}
& G_{0}=\sum\left|\alpha_{j}\right|^{2}[\left.r_{j j}(t, x, k)+u_{j j}(t, x, k)\right] \\
&=\frac{1}{(2 \pi)^{d}} \sum\left|\alpha_{j}\right|^{2}\left[\frac{\bar{W}\left(k+p_{j}\right)-\bar{W}(k)}{\theta\left(1-\hat{\chi}\left(p_{j}\right)\right)+i\left(k+\frac{p_{j}}{2}\right) \cdot p_{j}}+\frac{\bar{W}\left(k-p_{j}\right)-\bar{W}(k)}{\theta\left(1-\hat{\chi}\left(p_{j}\right)\right)+i\left(k-\frac{p_{j}}{2}\right) \cdot p_{j}}\right. \\
&\left.\quad+\frac{\bar{W}\left(k-p_{j}\right)-\bar{W}(k)}{\theta\left(1-\hat{\chi}\left(p_{j}\right)\right)-i\left(k-\frac{p_{j}}{2}\right) \cdot p_{j}}+\frac{\bar{W}\left(k+p_{j}\right)-\bar{W}(k)}{\theta\left(1-\hat{\chi}\left(p_{j}\right)\right)-i\left(k+\frac{p_{j}}{2}\right) \cdot p_{j}}\right] \\
& \quad \sum\left|\alpha_{j}\right|^{2}\left\{K_{\theta}\left(k, p_{j}\right)\left[\bar{W}\left(k+p_{j}\right)-\bar{W}(k)\right]+K_{\theta}\left(k,-p_{j}\right)\left[\bar{W}\left(k-p_{j}\right)-\bar{W}(k)\right]\right\} .
\end{aligned}
$$

We used the radial symmetry of the function $\chi$ in the calculation above. The scattering kernel in (3.15) is given by

$$
\begin{aligned}
K_{\theta}(k, p) & =\frac{1}{(2 \pi)^{d}}\left[\frac{1}{\theta(1-\hat{\chi}(p))+i\left(k+\frac{p}{2}\right) \cdot p}+\frac{1}{\theta(1-\hat{\chi}(p))-i\left(k+\frac{p}{2}\right) \cdot p}\right] \\
& =\frac{1}{(2 \pi)^{d}} \frac{2 \theta(1-\hat{\chi}(p))}{\theta^{2}(1-\hat{\chi}(p))^{2}+\left(\left(k+\frac{p}{2}\right) \cdot p\right)^{2}}
\end{aligned}
$$

and coincides with that in (2.8).

Equation (3.8) implies that

$$
\frac{\partial \bar{W}}{\partial t}+k \cdot \nabla_{x} \bar{W}=G_{0}(t, x, k) .
$$

Thus, using (3.15) and (3.15), we obtain the following kinetic equation for $\bar{W}(t, x, k)$ :

$$
\begin{array}{r}
\frac{\partial \bar{W}}{\partial t}+k \cdot \nabla_{x} \bar{W}=\sum_{j=1}^{\infty}\left|\alpha_{j}\right|^{2}\left\{K_{\theta}\left(k, p_{j}\right)\left[\bar{W}\left(k+p_{j}\right)-\bar{W}(k)\right]\right. \\
\left.+K_{\theta}\left(k,-p_{j}\right)\left[\bar{W}\left(k-p_{j}\right)-\bar{W}(k)\right]\right\}
\end{array}
$$

which is nothing but (2.9). 
Bounds for the discrete kinetic equation. In order for the solution $\bar{W}$ of (3.16) to satisfy a uniform bound in $L^{2}$ we need the total scattering cross-section

$$
S(k)=\sum\left|\alpha_{j}\right|^{2}\left\{K_{\theta}\left(k, p_{j}\right)+K_{\theta}\left(k,-p_{j}\right)\right\}
$$

to belong to $L^{\infty}\left(\mathbb{R}^{d}\right)$. This condition is ensured, for instance, under assumption (2.5) in Theorem 2.1. In order to show that (3.16) is, indeed, dissipative in the $L^{2}$-norm, we note that the kernel $K_{\theta}(k, p)$ has the property

$$
K_{\theta}(k-p, p)=K_{\theta}(k,-p) .
$$

This symmetry relation is a discrete version of the usual symmetry property $\sigma(k, p)=$ $\sigma(p, k)$ of the differential scattering cross-section in the transport theory. Indeed, multiplying (3.16) by $\bar{W}$, using again the convention $p_{-j}=-p_{j}, \alpha_{-j}=\alpha_{j}$ and integrating we get

$$
\begin{aligned}
& \frac{d}{d t}\|\bar{W}(t)\|_{L^{2}\left(\mathbb{R}^{2 d}\right)}^{2} \\
& =2 \sum_{j \in \mathbb{Z}}\left|\alpha_{j}\right|^{2} \int K_{\theta}\left(k, p_{j}\right)\left[\bar{W}\left(t, x, k+p_{j}\right)-\bar{W}(t, x, k)\right] \bar{W}(t, x, k) d k d x \\
& =2 \sum_{j \in \mathbb{Z}}\left|\alpha_{j}\right|^{2} \int K_{\theta}\left(k-p_{j}, p_{j}\right)\left[\bar{W}(t, x, k)-\bar{W}\left(t, x, k-p_{j}\right)\right] \bar{W}\left(t, x, k-p_{j}\right) d k d x \\
& =2 \sum_{j \in \mathbb{Z}}\left|\alpha_{j}\right|^{2} \int K_{\theta}\left(k,-p_{j}\right)\left[\bar{W}(t, x, k)-\bar{W}\left(t, x, k-p_{j}\right)\right] \bar{W}\left(t, x, k-p_{j}\right) d k d x \\
& =2 \sum_{j \in \mathbb{Z}}\left|\alpha_{j}\right|^{2} \int K_{\theta}\left(k, p_{j}\right)\left[\bar{W}(t, x, k)-\bar{W}\left(t, x, k+p_{j}\right)\right] \bar{W}\left(t, x, k+p_{j}\right) d k d x \\
& =-\sum_{j \in \mathbb{Z}}\left|\alpha_{j}\right|^{2} \int K_{\theta}\left(k,-p_{j}\right)\left[\bar{W}(t, x, k)-\bar{W}\left(t, x, k+p_{j}\right)\right]^{2} d k d x \leq 0 .
\end{aligned}
$$

We conclude that

$$
\|\bar{W}(t)\|_{L^{2}\left(\mathbb{R}^{2 d}\right)} \leq\left\|W_{0}(t)\right\|_{L^{2}\left(\mathbb{R}^{2 d}\right)} .
$$

Bounds for the second corrector. We go back to constructing and bounding the second corrector $W_{2}$. Equation (3.7) for $W_{2}$ may be now written as

$$
k \cdot \nabla_{z} W_{2}+\theta\left(W_{2}-\int \chi(y) W_{2}(t, x, z-y) d y\right)=\int e^{i p \cdot z} B(t, x, k, p) \frac{d p}{(2 \pi)^{d}}
$$

with

$$
\begin{aligned}
B(p)=G(p)- & G_{0} \delta(p) \\
= & \sum_{j, l} \alpha_{j} \alpha_{l}\left[s_{j l}(t, x, k) \delta\left(p-\left(p_{j}+p_{l}\right)\right)+q_{j l}(t, x, k) \delta\left(p+p_{j}+p_{l}\right)\right] \\
& \quad+\sum_{j \neq l} \alpha_{j} \alpha_{l}\left[r_{j l}(t, x, k) \delta\left(p-\left(p_{l}-p_{j}\right)+u_{j l}(t, x, k) \delta\left(p-\left(p_{j}-p_{l}\right)\right)\right] .\right.
\end{aligned}
$$

It is convenient to look first at a general equation of the form

$$
k \cdot \nabla_{z} w+\theta\left(w-\int \chi(y) w(t, x, z-y) d y\right)=g(t, x, k) e^{i \omega \cdot z}
$$


Its solution is given explicitly as

$$
w(t, x, z, k)=\frac{g(t, x, k) e^{i \omega \cdot z}}{\theta(1-\hat{\chi}(\omega))+i k \cdot \omega} .
$$

Hence, the $L^{2}$-norm of the function $w_{\varepsilon}(t, x, k)=w(t, x, x / \varepsilon, k)$ may be estimated as

$$
\left\|w_{\varepsilon}(t)\right\|_{L^{2}\left(\mathbb{R}^{2 d}\right)} \leq \frac{C}{\theta(1-\hat{\chi}(\omega))}\|g(t)\|_{L^{2}\left(\mathbb{R}^{2 d}\right)} .
$$

The explicit expressions (3.9)-(3.12) for the functions $s_{j l}, r_{j l}, q_{j l}$ and $u_{j l}$ imply that (we now use the notation $\|\ldots\|_{2}$ for $\|\ldots\|_{L^{2}\left(\mathbb{R}^{2 d}\right)}$ )

$$
\left\|s_{j l}(t)\right\|_{2}+\left\|r_{j l}(t)\right\|_{2}+\left\|q_{j l}(t)\right\|_{2}+\left\|u_{j l}(t)\right\|_{2} \leq C \frac{\| \bar{W}(t)}{\theta\left(1-\hat{\chi}\left(p_{l}\right)\right)} .
$$

The estimate (3.20), together with (3.21), the defining equation (3.19) for $W_{2}$, expression (3.13) for $G(p)$, and the uniform bound (3.18) on $\|\bar{W}(t)\|_{L^{2}\left(\mathbb{R}^{2 d}\right)}$, imply that

$$
\begin{aligned}
\left\|W_{2}(t)\right\|_{2} & \leq \frac{C}{\theta^{2}}\left(\sum_{j, l=1}^{\infty} \frac{\left|\alpha_{j}\right|\left|\alpha_{l}\right|}{\left(1-\hat{\chi}\left(p_{l}\right)\right)\left(1-\hat{\chi}\left(p_{j}+p_{l}\right)\right)}+\sum_{j \neq l} \frac{\left|\alpha_{j}\right|\left|\alpha_{l}\right|}{\left(1-\hat{\chi}\left(p_{l}\right)\right)\left(1-\hat{\chi}\left(p_{j}-p_{l}\right)\right)}\right) \\
& \leq \frac{C}{\theta^{2}},
\end{aligned}
$$

provided that

$$
\sum_{j, l=1}^{\infty} \frac{\left|\alpha_{j}\right|\left|\alpha_{l}\right|}{\left(1-\hat{\chi}\left(p_{l}\right)\right)\left(1-\hat{\chi}\left(p_{j}+p_{l}\right)\right)}+\sum_{j \neq l} \frac{\left|\alpha_{j}\right|\left|\alpha_{l}\right|}{\left(1-\hat{\chi}\left(p_{l}\right)\right)\left(1-\hat{\chi}\left(p_{j}-p_{l}\right)\right)}<+\infty
$$

which is the assumptions (2.6) in Theorem 2.1 on the sequence $\alpha_{j}$.

4. Proof of Theorem 2.1: estimates on the remainder. Now, that we have shown that both $W_{1}^{\varepsilon}$ and $W_{2}^{\varepsilon}$ are uniformly bounded in $L^{2}$, it remains only to verify that the remainder $R_{\varepsilon}$ in the expansion (3.1) is small. Recall that

$$
R_{\varepsilon}=W_{\varepsilon}-\bar{W}-\sqrt{\varepsilon} W_{1}^{\varepsilon}-\varepsilon W_{2}^{\varepsilon} .
$$

Equation (2.1) may be expanded as

$$
\begin{aligned}
& \frac{\partial W_{0}}{\partial t}+\sqrt{\varepsilon} \frac{\partial W_{1}}{\partial t}+\varepsilon \frac{\partial W_{2}}{\partial t}+\frac{\partial R_{\varepsilon}}{\partial t}+k \cdot \nabla_{x} W_{0}+\sqrt{\varepsilon} k \cdot \nabla_{x} W_{1}+\varepsilon k \cdot \nabla_{x} W_{2}+k \cdot \nabla_{x} R_{\varepsilon} \\
& +\frac{1}{\sqrt{\varepsilon}} k \cdot \nabla_{z} W_{1}+k \cdot \nabla_{z} W_{2}+\frac{\theta}{\varepsilon}\left(W_{\varepsilon}-\chi_{\varepsilon} \star W_{\varepsilon}\right)=\frac{1}{\sqrt{\varepsilon}} \mathcal{L}_{\varepsilon} W_{0}+\mathcal{L}_{\varepsilon} W_{1}+\sqrt{\varepsilon} \mathcal{L}_{\varepsilon} W_{2}+\mathcal{L}_{\varepsilon} R_{\varepsilon} .
\end{aligned}
$$

Here we have used the operator $\mathcal{L}_{\varepsilon}$ defined in equation (2.2). We re-write (4.1) as

$$
\frac{\partial R_{\varepsilon}}{\partial t}+k \cdot \nabla_{x} R_{\varepsilon}+\frac{\theta}{\varepsilon}\left(R_{\varepsilon}-\chi_{\varepsilon} \star R_{\varepsilon}\right)-\mathcal{L}_{\varepsilon} R_{\varepsilon}=g_{\varepsilon}
$$

with

$$
\begin{aligned}
g_{\varepsilon}(t, x, k)= & -\frac{\partial \bar{W}}{\partial t}-\sqrt{\varepsilon} \frac{\partial W_{1}}{\partial t}-\varepsilon \frac{\partial W_{2}}{\partial t}-k \cdot \nabla_{x} \bar{W}-\sqrt{\varepsilon} k \cdot \nabla_{x} W_{1}-\varepsilon k \cdot \nabla_{x} W_{2} \\
& -\frac{1}{\sqrt{\varepsilon}} k \cdot \nabla_{z} W_{1}-k \cdot \nabla_{z} W_{2}+\frac{1}{\sqrt{\varepsilon}} \mathcal{L}_{\varepsilon} \bar{W}+\mathcal{L}_{\varepsilon} W_{1}+\sqrt{\varepsilon} \mathcal{L}_{\varepsilon} W_{2} \\
& -\frac{\theta}{\varepsilon}\left(\bar{W}+\sqrt{\varepsilon} W_{1}+\varepsilon W_{2}-\chi_{\varepsilon} \star\left(\bar{W}+\sqrt{\varepsilon} W_{1}+\varepsilon W_{2}\right)\right) .
\end{aligned}
$$


We will show that

$$
\left\|g_{\varepsilon}(t)\right\|_{L^{2}\left(\mathbb{R}^{2 d}\right)} \leq C_{T} \sqrt{\varepsilon} .
$$

This will imply that $\left\|R_{\varepsilon}(t)\right\| \leq C_{T} \sqrt{\varepsilon}$ for $0 \leq t \leq T$ and finish the proof of Theorem 2.1 .

As a general remark, we will use throughout the proof that $D_{x}^{l} \bar{W}$ satisfies the equation (2.1) and therefore

$$
\left\|D_{x}^{l} \bar{W}(t)\right\|_{L^{2}\left(\mathbb{R}^{2 d}\right)} \leq\left\|D_{x}^{l} \bar{W}^{0}\right\|_{L^{2}\left(\mathbb{R}^{2 d}\right)} .
$$

Let us look at the term on the last line in (4.3):

$$
\bar{W}+\sqrt{\varepsilon} W_{1}+\varepsilon W_{2}-\chi_{\varepsilon} \star\left(\bar{W}+\sqrt{\varepsilon} W_{1}+\varepsilon W_{2}\right)=I_{0}^{\varepsilon}+\sqrt{\varepsilon} I_{1}^{\varepsilon}+\varepsilon I_{2}^{\varepsilon}
$$

The first right hand side term above is

$$
I_{0}^{\varepsilon}(t, x, k)=\bar{W}-\chi_{\varepsilon} \star \bar{W} .
$$

After Fourier transform, its $L^{2}$-norm is estimated as

$$
\left\|I_{0}^{\varepsilon}\right\|_{2}^{2}=\int|1-\hat{\chi}(\varepsilon p)|^{2}|\widehat{\bar{W}}(p, y)|^{2} \frac{d p d y}{(2 \pi)^{2 d}} .
$$

Note that the radial symmetry of $\chi(x)$ implies that $\hat{\chi}^{\prime}(0)=0$ and thus

$$
|1-\hat{\chi}(p)| \leq C|p|^{2} .
$$

This leads to

$$
\left\|I_{0}^{\varepsilon}\right\|_{2}^{2} \leq C \varepsilon^{4} \int|p|^{4}|\widehat{\bar{W}}(p, y)|^{2} d p d y \leq C \varepsilon^{4}\left\|D^{2} \bar{W}\right\|_{2} \leq C \varepsilon^{4},
$$

as the initial data $W_{0}$ for the kinetic equation (3.16) is smooth. The second term in $(4.5)$ is

$$
\begin{aligned}
I_{1}^{\varepsilon}(t, x, k) & =W_{1}^{\varepsilon}-\chi_{\varepsilon} \star W_{1}^{\varepsilon}=\int \chi(y)\left[W_{1}\left(x, \frac{x}{\varepsilon}\right)-W_{1}\left(x-\varepsilon y, \frac{x}{\varepsilon}-y\right)\right] d y \\
& =\int \chi(y)\left[W_{1}\left(x, \frac{x}{\varepsilon}\right)-W_{1}\left(x, \frac{x}{\varepsilon}-y\right)+W_{1}\left(x, \frac{x}{\varepsilon}-y\right)-W_{1}\left(x-\varepsilon y, \frac{x}{\varepsilon}-y\right)\right] d y \\
& =I_{11}^{\varepsilon}+I_{12}^{\varepsilon} .
\end{aligned}
$$

The term

$$
I_{11}^{\varepsilon}=\chi(z) \star_{z} W_{1}^{\varepsilon}\left(t, x, z=\frac{x}{\varepsilon}, k\right)
$$

is not small and will be used to cancel the term $k \cdot \nabla_{z} W_{1}$ in (4.3), using the defining equation for $W_{1}$. We check that the other one is small:

$$
I_{12}^{\varepsilon}(x, k)=\int \chi(y)\left[W_{1}\left(x, \frac{x}{\varepsilon}-y, k\right)-W_{1}\left(x-\varepsilon y, \frac{x}{\varepsilon}-y, k\right)\right] d y .
$$


Observe that, using (3.4),

$$
\begin{aligned}
\left\|I_{12}^{\varepsilon}(x, k)\right\|_{2} \leq & \int \chi(y)\left\|W_{1}\left(x, \frac{x}{\varepsilon}-y, k\right)-W_{1}\left(x-\varepsilon y, \frac{x}{\varepsilon}-y, k\right)\right\|_{2} d y \\
\leq & \int \chi(y)\left\|W_{1}\left(x+\varepsilon y, \frac{x}{\varepsilon}, k\right)-W_{1}\left(x, \frac{x}{\varepsilon}, k\right)\right\|_{2} d y \\
\leq & \int \chi(y) \| \int e^{i p \cdot x / \varepsilon} \frac{\hat{V}(p)}{i[(\theta(1-\hat{\chi}(p))+i k \cdot]}\left[\bar{W}_{1}\left(t, x+\varepsilon y, k-\frac{p}{2}\right)-\right. \\
& \left.\bar{W}_{1}\left(t, x, k-\frac{p}{2}\right)+\bar{W}_{1}\left(t, x, k+\frac{p}{2}\right)-\bar{W}_{1}\left(t, x+\varepsilon y, k+\frac{p}{2}\right)\right] \frac{d p}{(2 \pi)^{d}} \|_{2} d y \\
\leq & \frac{2}{\theta} \int \frac{|\hat{V}(p)|}{1-\hat{\chi}(p)} \frac{d p}{(2 \pi)^{d}} \int \chi(y)\left\|\bar{W}_{1}(t, x, k)-\bar{W}_{1}(t, x+\varepsilon y, k)\right\|_{2} d y \\
\leq & \frac{C \varepsilon}{\theta} \int|y| \chi(y) d y\left\|\nabla_{x} \bar{W}(t)\right\|_{2} .
\end{aligned}
$$

We used above the assumption (2.5), and thus

$$
\left\|I_{12}^{\varepsilon}\right\|_{2} \leq C \varepsilon .
$$

Next, we bound

$$
\begin{aligned}
I_{2}^{\varepsilon}(t, x, k) & =W_{2}^{\varepsilon}-\chi_{\varepsilon} \star W_{2}^{\varepsilon}=\int \chi(y)\left[W_{2}\left(x, \frac{x}{\varepsilon}\right)-W_{2}\left(x-\varepsilon y, \frac{x}{\varepsilon}-y\right)\right] d y \\
& =\int \chi(y)\left[W_{2}\left(x, \frac{x}{\varepsilon}\right)-W_{2}\left(x, \frac{x}{\varepsilon}-y\right)+W_{2}\left(x, \frac{x}{\varepsilon}-y\right)-W_{2}\left(x-\varepsilon y, \frac{x}{\varepsilon}-y\right)\right] d y \\
& =I_{21}^{\varepsilon}+I_{22}^{\varepsilon} .
\end{aligned}
$$

The term $I_{21}$ is not small and will be used to cancel the term $k \cdot \nabla_{z} W_{2}$ in (4.3), using the defining equation for $W_{2}$. We check that the other one is small:

$$
I_{22}^{\varepsilon}(t, x, k)=\int \chi(y)\left[W_{2}\left(x, \frac{x}{\varepsilon}-y\right)-W_{2}\left(x-\varepsilon y, \frac{x}{\varepsilon}-y\right)\right] d y .
$$

Once again,

$$
\left|W_{2}(x, z)-W_{2}(x-\varepsilon y, z)\right| \leq \int\left|\hat{W}_{2}(x, p)-\hat{W}_{2}(x-\varepsilon y, p)\right| d p .
$$

The function $W_{2}$ has many similar terms that come from (3.13), we look at the one that has the function $s_{j l}(t, x, k)$ in it:

$$
\hat{W}_{2}^{s}(t, x, p, k)=\sum \frac{\alpha_{j} \alpha_{l}\left[s_{j l}(t, x, k) \delta\left(p-\left(p_{j}+p_{l}\right)\right)\right.}{\theta\left(1-\hat{\chi}\left(p_{j}+p_{l}\right)\right)+i k \cdot\left(p_{j}+p_{l}\right)}
$$

The corresponding term in $(4.11)$ is

$$
\begin{aligned}
\left|W_{2}^{s}(x, z)-W_{2}^{s}(x-\varepsilon y, z)\right| & \leq \int\left|\hat{W}_{2}^{s}(x, p)-\hat{W}_{2}^{s}(x-\varepsilon y, p)\right| d p \\
& \leq \frac{C}{\theta} \sum \frac{\left|\alpha_{j} \alpha_{l}\right|}{1-\hat{\chi}\left(p_{j}+p_{l}\right)}\left|s_{j l}(t, x, k)-s_{j l}(t, x-\varepsilon y, k)\right| .
\end{aligned}
$$


This leads to a term in term in $I_{22}^{\varepsilon}$ that is bounded as

$$
\begin{aligned}
\left|I_{22}^{s}(t, x, k)\right| & \leq \int \chi(y)\left|W_{2}^{s}\left(x, \frac{x}{\varepsilon}-y\right)-W_{2}^{s}\left(x-\varepsilon y, \frac{x}{\varepsilon}-y\right)\right| d y \\
& \leq \frac{C}{\theta} \sum \frac{\left|\alpha_{j} \alpha_{l}\right|}{1-\hat{\chi}\left(p_{j}+p_{l}\right)} \int \chi(y)\left|s_{j l}(t, x, k)-s_{j l}(t, x-\varepsilon y, k)\right| d y .
\end{aligned}
$$

Its $L^{2}$-norm is estimated as

$$
\begin{aligned}
\left\|\mid I_{22}^{2}(t)\right\|_{2} & \leq \frac{C}{\theta} \sum \frac{\mid \alpha_{j} \alpha_{l}}{\left(1-\hat{\chi}\left(p_{j}+p_{l}\right)\right)} \int \chi(y)\left\|s_{j l}(t, x, k)-s_{j l}(t, x-\varepsilon y, k)\right\|_{2} d y \\
& \leq \frac{C \varepsilon}{\theta} \sum \frac{\left|\alpha_{j} \alpha_{l}\right|}{1-\hat{\chi}\left(p_{j}+p_{l}\right)} \int|y| \chi(y) d y \sup _{j, l}\left\|\nabla_{x} s_{j l}(t)\right\|_{2} \\
& \leq \frac{C \varepsilon}{\theta} \sum \frac{\left|\alpha_{j} \alpha_{l}\right|}{\left(1-\hat{\chi}\left(p_{j}+p_{l}\right)\right)\left(1-\hat{\chi}\left(p_{l}\right)\right)} \int|y| \chi(y) d y\left\|\nabla_{x} \bar{W}(t)\right\|_{2} \leq C \varepsilon .
\end{aligned}
$$

The other contributions in $I_{22}$ that arise from the terms in $W_{2}$ that involve the functions $r_{j l}, q_{j l}$ and $u_{j l}$ may be bounded in an identical way, so that

$$
\left\|I_{22}\right\|_{2} \leq C \varepsilon
$$

as well.

Equations (3.2) and (3.5) for $W_{1}$ and $W_{2}$, respectively, imply that the error term $g_{\varepsilon}$ given by (4.3) may be written as

$$
\begin{aligned}
g_{\varepsilon}(t, x, k)= & -\sqrt{\varepsilon} \frac{\partial W_{1}}{\partial t}-\varepsilon \frac{\partial W_{2}}{\partial t}-\sqrt{\varepsilon} k \cdot \nabla_{x} W_{1}-\varepsilon k \cdot \nabla_{x} W_{2} \\
& +\sqrt{\varepsilon} \mathcal{L}_{\varepsilon} W_{2}-\frac{\theta}{\varepsilon}\left(I_{0}+\sqrt{\varepsilon} I_{12}+\varepsilon I_{22}\right)
\end{aligned}
$$

with $I_{0}, I_{12}$ and $I_{22}$ defined by (4.6), (4.8) and (4.10).

Since $\frac{\partial \bar{W}}{\partial t}$ satisfies the same equation as $\bar{W}$, we also have

$$
\left\|\frac{\partial \bar{W}(t)}{\partial t}\right\|_{L^{2}\left(\mathbb{R}^{2 d}\right)} \leq\left\|\frac{\partial \bar{W}_{0}}{\partial t}\right\|_{L^{2}\left(\mathbb{R}^{2 d}\right)}, \quad \forall t \geq 0 .
$$

Then, the operator $\mathcal{K}_{\theta}^{\delta}$ being bounded in $L^{2}$, we deduce from the equation on $\bar{W}$ that

$$
\left\|k \cdot \nabla_{x} \bar{W}\right\|_{L^{2}\left(\mathbb{R}^{2 d}\right)} \leq\left\|\frac{\partial \bar{W}(t)}{\partial t}\right\|_{L^{2}\left(\mathbb{R}^{2 d}\right)}+\left\|\mathcal{K}_{\theta}^{\delta} \bar{W}(t)\right\|_{L^{2}\left(\mathbb{R}^{2 d}\right)} \leq C
$$

where $C$ involves $\left\|\bar{W}_{0}\right\|_{L^{2}\left(\mathbb{R}^{2 d}\right)}$ and $\left\|k \cdot \nabla W_{0}\right\|_{L^{2}\left(\mathbb{R}^{2 d}\right)}$. Therefore one may verify in a direct manner that

$$
\left\|\frac{\partial W_{1}}{\partial t}\right\|_{2}+\left\|\frac{\partial W_{2}}{\partial t}\right\|_{2}+\left\|k \cdot \nabla_{x} W_{1}\right\|_{2}+\left\|k \cdot \nabla_{x} W_{2}\right\|_{2} \leq C
$$

using the definition of $W_{1}$ and $W_{2}$. The uniform boundedness of $\mathcal{L}^{\varepsilon}$ in $L^{2}$ implies that $\left\|\mathcal{L}_{\varepsilon} W_{2}\right\|_{2} \leq C$. Altogether, these bounds, taken with the estimates (4.7), (4.9) and (4.12), imply (4.4). Therefore, $\left\|R_{\varepsilon}(t)\right\| \leq C \sqrt{\varepsilon}$ and the proof of Theorem 2.1 is complete for a smooth initial data. Passing to a pure $L^{2}$ statement is automatic by density and $L^{2}$ stability. 
5. Proof of Theorems 2.2 and 2.3. The proofs of the last two Theorems follow similar lines and thus we only treat Theorem 2.3 which involves more elaborate estimates.

The main difficulty is to derive the $L^{2}$ bounds on the operator $\mathcal{P}_{\theta}$. We recall that, once this is done, the existence of a unique solutions in $C\left([0, \infty) ; L^{2}\left(\mathbb{R}^{d}\right)\right)$ is standard using the Banach fixed point Theorem. This theory provides a solution both for $\theta>0$ or $\theta=0$ with the corresponding assumptions.

The asymptotic behavior as $\theta \rightarrow 0$ is also standard and we recall the argument without details. It follows from the inequalities

$$
\begin{aligned}
\left\|\mathcal{P}_{\theta} \bar{U}_{\theta}-\mathcal{P}_{0} \bar{Z}\right\|_{L^{2}\left(\mathbb{R}^{d}\right)} & \leq\left\|\mathcal{P}_{\theta}\left[\bar{U}_{\theta}-\bar{Z}\right]\right\|_{L^{2}\left(\mathbb{R}^{d}\right)}+\left\|\left[\mathcal{P}_{0}-\mathcal{P}_{\theta}\right] \bar{Z}\right\|_{L^{2}\left(\mathbb{R}^{d}\right)} \\
& \leq C\left\|\bar{U}_{\theta}-\bar{Z}\right\|_{L^{2}\left(\mathbb{R}^{d}\right)}+o(\theta) .
\end{aligned}
$$

Indeed, since we have

$$
\frac{\partial\left(\bar{U}_{\theta}-\bar{Z}\right)}{\partial t}+k \cdot \nabla_{x}\left[\bar{U}_{\theta}-\bar{Z}\right]=\mathcal{P}_{\theta} \bar{U}_{\theta}-\mathcal{P}_{0} \bar{Z}
$$

we deduce

$$
\begin{aligned}
\frac{1}{2} \frac{d}{d t}\left\|\bar{U}_{\theta}(t)-\bar{Z}(t)\right\|_{L^{2}\left(\mathbb{R}^{2 d}\right)}^{2} & \leq\left\|\bar{U}_{\theta}(t)-\bar{Z}(t)\right\|_{L^{2}\left(\mathbb{R}^{2 d}\right)}\left\|\mathcal{P}_{\theta} \bar{U}_{\theta}-\mathcal{P}_{0} \bar{Z}\right\|_{L^{2}\left(\mathbb{R}^{d}\right)} \\
& \leq\left\|\bar{U}_{\theta}(t)-\bar{Z}(t)\right\|_{L^{2}\left(\mathbb{R}^{2 d}\right)}\left[C\left\|\bar{U}_{\theta}-\bar{Z}\right\|_{L^{2}\left(\mathbb{R}^{2 d}\right)}+o(\theta)\right] .
\end{aligned}
$$

Then, thanks to the Gronwall lemma, we conclude that the $L^{2}$ limit of $\bar{U}_{\theta}(t)$ is $\bar{Z}(t)$.

It remains to prove the $L^{2}$ bounds on the operator $\mathcal{P}_{\theta}$ which we state in the

LEMma 5.1. With the assumption (2.14) and with

$$
M_{\chi}=\int_{0}^{\infty}\left[\frac{1-\hat{\chi}(r)}{r^{2}}+\frac{\left|\hat{\chi}^{\prime}(r)\right|}{r}\right]^{2} \frac{r d r}{1-\hat{\chi}(r)}<\infty
$$

we have

$$
\left\|\mathcal{P}_{\theta} U\right\|_{L^{2}\left(\mathbb{R}^{d}\right)} \leq C\left(M_{\alpha}+\theta M_{\chi}\right)
$$

Notice that the integrability assumption $M_{\chi}<\infty$ involves a further cancellation of second derivatives $\hat{\chi}^{\prime \prime}(0)=0$.

Proof. We consider a test function $V \in L^{2}\left(\mathbb{R}^{d}\right)$ and write

$$
\begin{aligned}
& \int \mathcal{P}_{\theta}[U](k) V(k) d k=\int \alpha(p-k) \frac{2 \theta(1-\hat{\chi}(p-k))}{\theta^{2}(1-\hat{\chi}(p-k))^{2}+\left(\frac{(p+k) \cdot(p-k)}{2}\right)^{2}}[U(p)-U(k)] V(k) \frac{d p d k}{(2 \pi)^{d}} \\
& =C \iint \frac{2 \alpha\left(q_{-}\right) \theta\left(1-\hat{\chi}\left(q_{-}\right)\right)}{\theta^{2}\left(1-\hat{\chi}\left(q_{-}\right)\right)^{2}+\left(\frac{q_{+} \cdot q_{-}}{2}\right)^{2}}\left[U\left(\frac{q_{+}+q_{-}}{2}\right)-U\left(\frac{q_{+}-q_{-}}{2}\right)\right] V\left(\frac{q_{+}-q_{-}}{2}\right) d q_{+} d q_{-}
\end{aligned}
$$

This involves two terms which can be treated in a similar manner and thus we only consider the "cross term" $U\left(\frac{q_{+}+q_{-}}{2}\right) V\left(\frac{q_{+}-q_{-}}{2}\right)$. 
We set $q_{-}=r \omega$ and $q_{+}=q_{1} \omega+q^{\prime}$ with $q_{1} \in \mathbb{R}$ and $q^{\prime} \perp \omega$, and $\varphi(r)=1-\hat{\chi}\left(q_{-}\right)$. We arrive at

$C \iint \alpha(r \omega) \frac{2 \theta \varphi(r)}{(\theta \varphi(r))^{2}+\left(\frac{q_{1} r}{2}\right)^{2}} U\left(q^{\prime}+\left(q_{1}+r\right) \omega\right) V\left(q^{\prime}+\left(q_{1}-r\right) \omega\right) d q_{1} d q^{\prime} r^{d-1} d r d \omega$

$\leq C \iint \sup _{\rho>0}\left[\rho^{d-2} \alpha(\rho \omega)\right] \frac{2}{1+\frac{s^{2}}{2}} U\left(q^{\prime}+\omega\left(s \theta \frac{\varphi(r)}{r}+r\right)\right) V\left(q^{\prime}+\omega\left(s \theta \frac{\varphi(r)}{r}-r\right)\right) d q^{\prime} d s d r d \omega$

$=I+I I$,

where the integral is treated in two different ways for $s \theta|Q(r)| \leq \frac{1}{2}$ and $s \theta|Q(r)| \geq \frac{1}{2}$ with $Q(r)=\frac{\varphi(r)}{r^{2}}-\frac{\varphi^{\prime}(r)}{r}$.

On one hand, we have for $s \theta|Q(r)| \leq \frac{1}{2}$ :

$$
\begin{aligned}
I \leq C \int_{\omega, s} \sup _{\rho>0}\left[\rho^{d-2} \alpha(\rho \omega)\right] \frac{2}{1+\frac{s^{2}}{2}}\left(\int U^{2}\left(q^{\prime}+\omega\left(s \theta \frac{\varphi(r)}{r}+r\right)\right) d q^{\prime} d r\right. \\
\left.\quad \times \int V^{2}\left(q^{\prime}+\omega\left(s \theta \frac{\varphi(r)}{r}-r\right)\right) d q^{\prime} d r\right)^{1 / 2} d s d \omega \\
\leq C \int_{\omega, s} \sup _{\rho>0}\left[\rho^{d-2} \alpha(\rho \omega)\right] \frac{2}{1+\frac{s^{2}}{2}}\left(\int U^{2}\left(q^{\prime}+\omega R\right)(1-s \theta Q(r))^{-1} d q^{\prime} d R\right. \\
\left.\quad \times \int V^{2}\left(q^{\prime}+\omega R\right)(1+s \theta Q(r))^{-1} d q^{\prime} d R\right)^{1 / 2} d s d \omega \\
\leq C M_{\alpha}\|U\|_{L^{2}\left(\mathbb{R}^{d}\right)}\|V\|_{L^{2}\left(\mathbb{R}^{d}\right)},
\end{aligned}
$$

where we have used the change of variable $r \rightarrow R=r \pm s \theta \frac{\varphi(r)}{r}$.

On the other hand, we have for $s \theta|Q(r)| \geq \frac{1}{2}$ :

$$
\begin{aligned}
I I & \leq \tilde{C} \int_{\omega, r} \sup _{\rho>0}\left[\rho^{d-2} \alpha(\rho \omega)\right](\theta|Q(r)|)^{2}\left(\int U^{2}\left(q^{\prime}+\omega\left(s \theta \frac{\varphi(r)}{r}+r\right)\right) d q^{\prime} d s\right. \\
& \left.\int V^{2}\left(q^{\prime}+\omega\left(s \theta \frac{\varphi(r)}{r}-r\right)\right) d q^{\prime} d s\right)^{1 / 2} d r d \omega \\
& \leq \tilde{C} \int_{\omega, r} \sup _{\rho>0}\left[\rho^{d-2} \alpha(\rho \omega)\right](\theta|Q(r)|)^{2}\left(\int U^{2}\left(q^{\prime}+\omega S\right) d q^{\prime} d S\right. \\
& \left.\quad \int V^{2}\left(q^{\prime}+\omega S\right) d q^{\prime} d S\right)^{1 / 2} \frac{r d r}{\theta \varphi(r)} d \omega \\
& \leq C \theta M_{\chi}\|U\|_{L^{2}\left(\mathbb{R}^{d}\right)}\|V\|_{L^{2}\left(\mathbb{R}^{d}\right)},
\end{aligned}
$$

because

$$
\int(|Q(r)|)^{2} \frac{r d r}{\varphi(r)} \leq M_{\chi}<\infty
$$

This completes the proof of Lemma 5.1. 


\section{REFERENCES}

[1] G. Bal, A. Fannjiang, G. Papanicolaou and L. Ryzhik, Radiative transport in a periodic structure, Jour. Stat. Phys., 95 (1999), pp. 479-494.

[2] G. Bal, G. Papanicolaou, AND L. RYzhiK, Radiative transport limit for the random Schrödinger equations, Nonlinearity, 15 (2002), pp. 513-529.

[3] G. Bal, G. Papanicolaou, and L. Ryzhik, Self-averaging in time reversal for the parabolic wave equation, Stochastics and Dynamics, 4 (2002), pp. 507-531.

[4] A. Bensoussan, J.-L. Lions and G. Papanicolaou, Asymptotic analysis for periodic structures, North-Holland, New York, 1978.

[5] F. Castella, From the von Neumann equation to the quantum Boltzmann equation in a deterministic framework, Jour. Stat. Phys., 104 (2001), pp. 387-447.

[6] F. Castella, From the von Neumann equation to the quantum Boltzmann equation. II. Identifying the Born series, Jour. Stat. Phys., 106 (2002), pp. 1197-1220.

[7] F. CASTELla, On the derivation of a quantum Boltzmann equation from the periodic von Neumann equation, M2AN Math. Model. Numer. Anal., 33 (1999), pp. 329-349.

[8] F. Castella and A. Plagne, Non derivation of the quantum Boltzmann equation from the periodic von Neumann equation, Indiana Univ. Math. J., 51 (2002), pp. 963-1016.

[9] D. Cioranescu, P. Donato, An introduction to homogenization, Oxford Lectures Series in MAth. and Appl., 17, Oxford Univ. Press, 1999.

[10] L. ERDÖS AND H. T. YAU, Linear Boltzmann equation as the weak coupling limit of a random Schrödinger Equation, Comm. Pure Appl. Math., 53 (2000), pp. 667-735.

[11] A. Fannjiang, Self-Averaging Scaling Limits for Random Parabolic Waves, to appear in Arch. Rat. Mech., 2004.

[12] P. Gérard, P. A. Markowich, N. J. Mauser, and F. Poupaud, Homogenization limits and Wigner transforms, Comm. Pure Appl. Math., 50 (1997), pp. 323-380.

[13] T. Ho, L. Landau And A. Wilkins, On the weak coupling limit for a Fermi gas in a random potential, Rev. Math. Phys., 5 (1993), pp. 209-298.

[14] P.-L. Lions And T. Paul, Sur les mesures de Wigner, Rev. Mat. Iberoamericana, 9 (1993), pp. 553-618.

[15] F. NIER, Asymptotic analysis of a scaled Wigner equation and Quantum Scattering, Transp. Theor. Stat. Phys., 24 (1995), pp. 591-629.

[16] F. Poupaud And A. Vasseur, Classical and quantum transport in random media, J. Math. Pures Appl., 82 (2003), pp. 711-748.

[17] L. Ryzhik, G. Papanicolaou, and J. B. Keller, Transport equations for elastic and other waves in random media, Wave Motion, 24 (1996), pp. 327-370.

[18] H. Spohn, Derivation of the transport equation for electrons moving through random impurities, Jour. Stat. Phys., 17 (1977), pp. 385-412. 